University of Nebraska - Lincoln

DigitalCommons@University of Nebraska - Lincoln

Mechanical \& Materials Engineering Faculty

Publications

Mechanical \& Materials Engineering,

Department of

2004

\title{
Planetary Cliff Descent Using Cooperative Robots
}

\author{
Erik Mumm \\ University of Nebraska-Lincoln \\ Shane Farritor \\ University of Nebraska-Lincoln, sfarritor@unl.edu \\ Paola Pirjanian \\ Evolution Robotics, Pasadena, CA \\ Chris Leger \\ Jet Propulsion Laboratory, Pasadena, CA \\ Paul Schenker \\ Jet Propulsion Laboratory, Pasadena, CA
}

Follow this and additional works at: https://digitalcommons.unl.edu/mechengfacpub

Part of the Mechanical Engineering Commons

Mumm, Erik; Farritor, Shane; Pirjanian, Paola; Leger, Chris; and Schenker, Paul, "Planetary Cliff Descent Using Cooperative Robots" (2004). Mechanical \& Materials Engineering Faculty Publications. 22. https://digitalcommons.unl.edu/mechengfacpub/22

This Article is brought to you for free and open access by the Mechanical \& Materials Engineering, Department of at DigitalCommons@University of Nebraska - Lincoln. It has been accepted for inclusion in Mechanical \& Materials Engineering Faculty Publications by an authorized administrator of DigitalCommons@University of Nebraska Lincoln. 


\title{
Planetary Cliff Descent Using Cooperative Robots
}

\author{
ERIK MUMM AND SHANE FARRITOR \\ Department of Mechanical Engineering, University of Nebraska-Lincoln, NE 68588-0656, USA \\ sfarritor@unl.edu \\ PAOLO PIRJANIAN \\ Evolution Robotics, Pasadena, CA 91103, USA \\ CHRIS LEGER AND PAUL SCHENKER \\ Jet Propulsion Laboratory, Pasadena, CA 91109-8099, USA
}

\begin{abstract}
Future robotic planetary exploration will need to traverse geographically diverse and challenging terrain. Cliffs, ravines, and fissures are of great scientific interest because they may contain important data regarding past water flow and past life.

Highly sloped terrain is difficult and often impossible to safely navigate using a single robot. This paper describes a control system for a team of three robots that access cliff walls at inclines up to $70^{\circ}$. Two robot assistants, or anchors, lower a third robot, called the rappeller, down the cliff using tethers. The anchors use actively controlled winches to first assist the rappeller in navigation about the cliff face and then retreat to safe ground.

This paper describes the coordination of these three robots so they function as a team to explore the cliff face. Stability requirements for safe operation are identified and a behavior-based control scheme is presented. Behaviors are defined for the system and command fusion methods are described. Controller stability and sensitivity are examined. System performance is evaluated with simulation, a laboratory system, and testing in field environments.
\end{abstract}

Keywords: planetary exploration, robots for planetary exploration, cooperating robots, tethers, cliff decent, behavior

\section{Introduction}

Aggressive robotic exploration of Mars, or other extraterrestrial environments, requires the ability to traverse rough, unpredictable terrain. Future missions may use a system of cooperative robots to access challenging areas.

Cliffs, ravines, and fissures are of great scientific interest because they may contain important data regarding past water flows. Information about water is critical in searching for evidence of past life. Access to a cliff wall is of particular scientific interest because it allows observation of stratified geographic features. Current robotic technology is not applicable to extremely steep terrain. This paper presents a control method for a system of cooperating robots to access steep cliff walls.

The system used in this paper consists of a team of three robots to access cliffs at inclines up to $70^{\circ}$, Fig. 1 . Two robot anchors lower a third robot, called a rappeller, down the cliff using tethers. The anchors use actively controlled winches to assist the rappeller in navigating the cliff face. The rappeller is capable of omnidirectional (but non-holonomic) motion $(\theta=$ rappeller steering angle $\left.\left(-180^{\circ}<\theta<180^{\circ}\right)\right)$. The cliff slope is given by $\alpha$.

The tethers are attached to the rappeller and anchors robot via a 2 DOF gimbals, Fig. 2. The tether passes through outer and inner gimbals that are free to rotate. Sensors measure the tether angles with respect to the 


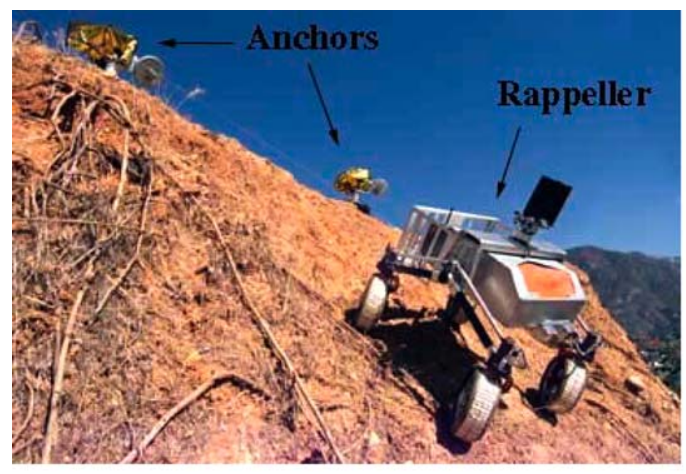

a) JPL Field System

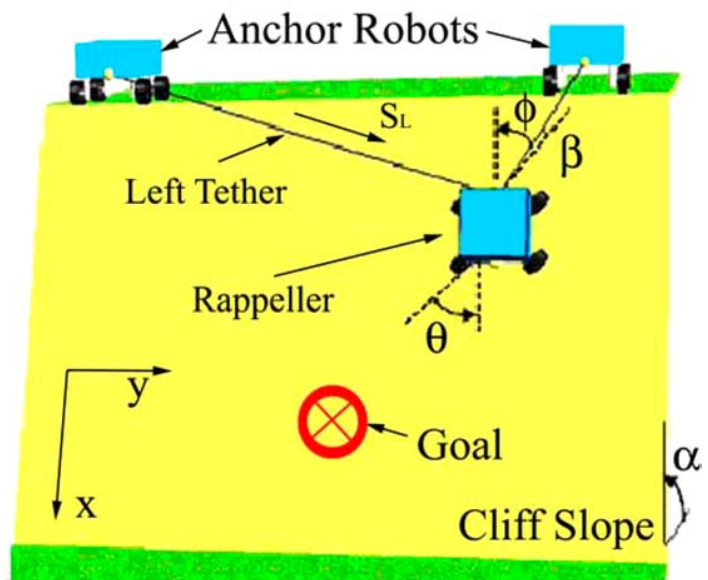

b) Schematic

Figure 1. Cliff decent robot team.

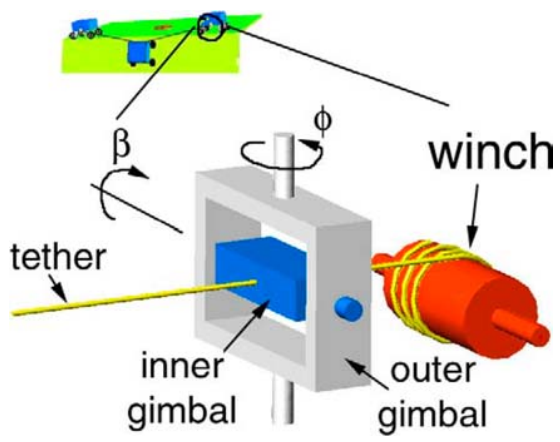

Figure 2. Tether gimbals and winch.

anchor robots ( $\phi$ and $\beta$ in Fig. 1(b) and Fig. 2) and inclinometers can be used to correct for changes in orientation of the anchors themselves. Similar gimbals are on the rappeller. The anchor robots are capable of independent motion and payout tether using a winch. Tether length $\left(S_{L} \& S_{R}\right)$ and velocity are controlled. It is assumed that the anchor robots know their position and communicate with RF transceivers (both are required for all autonomous planetary systems).

This paper describes a behavior-based control system that allows the robots to function as a team. Stability requirements for safe operation are identified. Behaviors are defined for the system of robots. A command fusion method is described that determines the resulting robot actions. The control architecture has been tested in both laboratory and field environments.

\section{Background}

Several techniques have been developed to autonomously operate mobile robots in unstructured environments. Deliberative, reactive and hybrid (deliberative and reactive combination) approaches have been used (Brooks, 1986; Khatib, 1986; Arkin, 1987, 1998; Latombe, 1991; Saffioti et al., 1997). Several methods have been developed for planetary exploration robots (Schenker et al., 2000a, 2000b; Pirjanian et al., 2000; Seraji et al., 2001; Weisbin et al., 1999; Gat et al., 1994; Farritor et al., 1998).

Reactive control approaches have advantages for planetary systems. Reactive controllers can often be implemented on systems with relatively limited computational ability since they only consider the current robot state and do not extensively plan future actions.

One reactive control approach, called behavior control (Arkin, 1998; Pirjanian, 2000), uses tightly coupled perception-action units referred to as behaviors. Each behavior uses appropriate sensor information to obtain a system objective (e.g. safe navigation, obstacle avoidance). Each behavior operates independently, cognizant only of its objective. Action selection mechanisms determine which behavior(s) contribute to system actions.

Behavior-based control has been used in many robot applications. It has been used in low-level obstacle avoidance for robotic manipulators (Khatib, 1986), and applied to mobile robots for obstacle avoidance, wall following, door traversal, and wandering (Brooks, 1986; Khatib, 1986; Arkin, 1987, 1998; Latombe, 1991; Murphy, 2000). Behavior control has also been implemented on space and underwater robots (Schenker et al., 2000a, 2000b; Rosenblatt et al., 2000; Gat et al., 1994).

Traditional mobile robots are not useful in steep terrain. Other work has studied navigating moderately sloped terrain using a kinematically reconfigurable rover (Schenker, 2000a). Access to steep terrain via 
a tethered rappeller robot has been demonstrated by DANTE II-a legged robot that rappelled into a volcanic crater (Apostolopoulos and Bares, 1995). DANTE II used a single fixed tether anchor to lower the robot into a volcano. With only one fixed tether the anchor could not assist the rappeller in motion across the cliff face. Any motion of the rappeller (or anchor) perpendicular to the tether produces an unstable configuration.

In this paper two tethers are attached to two robotic assistants that actively control their own position as well as tether payout. With mobile anchors the tether angles can be changed to increase the rappeller's mobility and safety.

Using multiple tethers has been studied in crane-like robots where multiple tethers (or cranes) cooperate to manipulate a passive mass in free space. Issues such as kinematics, dynamics, and optimal control have been studied (Shiang et al., 1999; Gorman et al., 2001). The cliff system differs because the manipulated mass (the rappeller) is active, the anchors are mobile, and the rappeller is constrained to motions on the cliff's face. Other work has studied tethers for electrical power and signal transmission (Fuskushima et al., 2001).

A behavior-based control system is implemented to coordinate the team of three robots (two anchors and one rappeller). The control system's software architecture considers robot communication, motion control, waypoint navigation, simple obstacle avoidance, and system safety (Pirjanian et al., 2000; Huntsberger et al., 2001).

\section{Stability Requirements}

Three stability requirements were defined for system safety: (1) Maintain tether tension, (2) Avoid unsafe tether configurations, and (3) Avoid tether singularities.

\subsection{Maintain Tether Tension}

Lack of tether tension could cause the rappeller to slip and swing freely. The steep slope helps ensure tether tension because the traction forces for the rappeller are greatly reduced (normal force $=m * g * \cos (\alpha)$ ). However, it is possible for the rappeller wheels to gain traction and create tether slack. Tether tension can be directly measured using load cells, or can be indirectly detected using tether angles and a system model $\left(\beta<0^{\circ}\right)$.

\subsection{Avoid Unsafe Tether Configurations}

Some tether configurations can inhibit rappeller motion or degrade navigation performance. For example, a tether caught on a rock cannot be coordinated with rappeller motion. In this case rappeller motion toward the goal is inhibited, rather than assisted, by the tether motion. Tether configurations are detected using tether angles and a system model.

\subsection{Avoid Tether Singularities}

Tether singularities can result in high tether forces causing unwanted motion (sliding) of the anchor robots, mechanical failure of the tether mechanism, or failure of the tether itself. For example, a tether singularity would occur if the rappeller robot moves vertically and approaches the midpoint between the anchors. Here the static tether force $\left(\frac{m g \sin (\alpha)}{2 \cos (\phi)}\right)$ would approach infinity $\left(\phi\right.$ approaches $\left.90^{\circ}\right)$. A different singularity will occur when the rappeller is directly below an anchor $(\phi=0)$.

\section{Coordinated Control}

A behavior-based control algorithm is used to determine the actions of each robot. Section 4.1 defines the system's behaviors. Section 4.2 describes the methods used to determine which behavior(s) dictate each robot's actions. Both command fusion and arbitration techniques are used.

\subsection{System Behaviors}

The system's behaviors and the interaction between them are shown in Fig. 3. The figure shows five levels with boxes denoting group behaviors and circles denoting single robot behaviors.

Waypoint Navigation is the highest behavior. This behavior commands the three robots to move the rappeller to a desired $\left(\underline{x}_{d}=\left[x_{d}, y_{d}\right]\right)$ location. The outputs of this behavior are velocity vectors for the rappeller robot, anchor robots, and tethers. The Communicate and Velocity Sync behaviors contribute to the Waypoint Navigation behavior.

Communicate shares sensor information at a predetermined ( $10 \mathrm{~Hz}$ field and laboratory experiments) rate between robots so each robot has complete knowledge of the system's state (sensor readings, desired potions, etc.). 


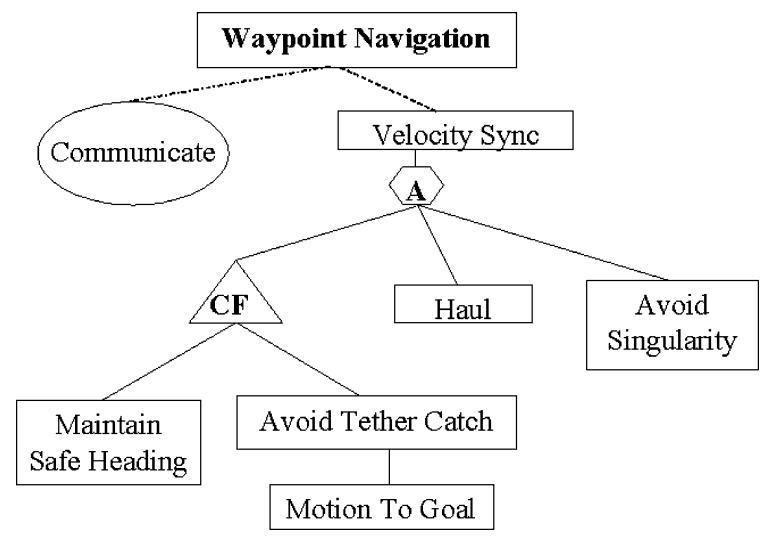

Figure 3. Behavioral hierarchy.

The Velocity Sync behavior produces a rappeller heading and coordinates movements of both tethers and anchor robots. The input to the Velocity Sync behavior is a safe rappeller heading dictated by lower level behaviors. The required tether winch velocities $\left(s_{i}\right)$ and anchor motions are determined by projecting the actual rappeller velocity (measured by averaging wheel encoders) onto the tether unit vector (1) (given by the tether angle sensors) where $\hat{i}$ and $\hat{j}$ are unit vectors in the $x$ and $y$ directions and the subscript $i$ corresponds to either the left or right tether. A low level controller is used on each anchor robot to maintain the desired winch speed $\left(s_{i}\right)$. This ensures the robots are coordinated.

$$
\begin{aligned}
\underline{T}_{i} & =\left[-\cos \left(\phi_{i}\right) \hat{i}+\sin \left(\phi_{i}\right) \hat{j}\right] \\
s_{i} & =\underline{V}_{\text {rappeller,actual }} \cdot \underline{T}_{i}
\end{aligned}
$$

This is a quassi-static control algorithm. This assumption is valid since dynamic forces created by rappeller motion have been shown to be less than $2 \%$ of static tether tension (Mumm, 2002). Slip of the rappeller wheels is generally not significant since the tether motion matches the rappeller motion (i.e. the motion would be coordinated even if the rappeller is on a frictionless cliff, or $\alpha=90^{\circ}$ ).

The Avoid Singularities behavior detects and avoids configuration singularities. Singularities are detected with simple system models (e.g., static tether force $\left.=\frac{m g \sin (\alpha)}{2 \cos (\phi)} \leq F_{\max }\right)$. The behavior produces a coordinated action to eliminate a tether singularity. For example, as described in Section 3, when the rappeller retreats up the cliff the tether angles become large and tether forces increase. In this situation, the Avoid

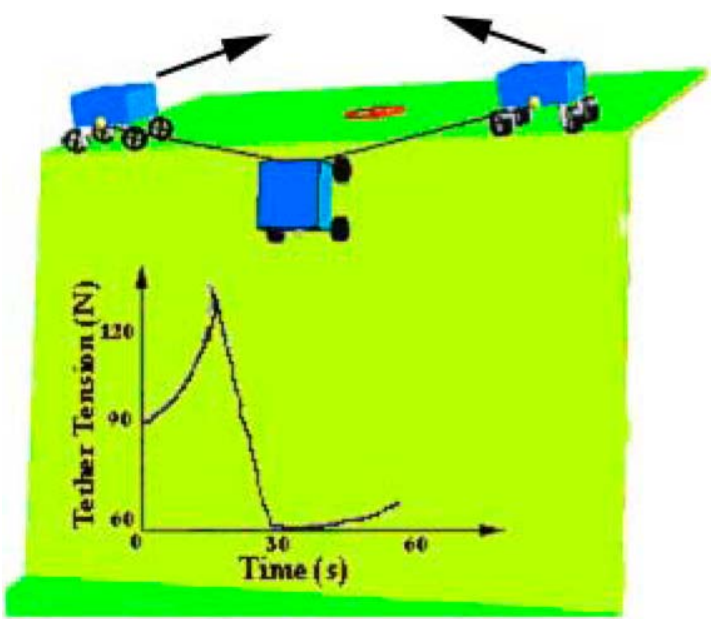

Figure 4. Anchor rovers retreat to eliminate singularity.

Singularities behavior commands the rappeller to halt and the anchors retreat. The anchors payout tether as they retreat to maintain the rappeller's position. These actions eliminate the singularities and the controller resumes normal operation. This situation is shown in Fig. 4 with arrows indicating the anchor velocities (and a superimposed graph of tether tension found with the simulation described below) during the maneuver.

The $\mathbf{H a u l}$ behavior uses the tethers to assist the rappeller rather than merely matching the rappeller motion. The haul behavior becomes active when the desired rappeller velocity is distinctly different from the actual rappeller velocity measured by its wheel sensors. This discrepancy can occur at low rappeller speeds when coulomb friction is significant, or when the rappeller contacts an obstacle and the wheels stall. The haul behavior uses the desired rappeller velocity rather than the measured velocity to determine the tether winch speeds (with a low pass filter to prevent jerk in the transition).

The Maintain Safe Heading behavior, on the third level, prevents the rappeller from choosing unsafe headings. Tether angles are used to determine the rappeller's position and a workspace map is used to determine the location of obstacles. The workspace map classifies areas as either safe or unsafe. Unsafe regions can include workspace constraints (rappeller cannot move outside the two anchors), be identified with a priori knowledge or identified with rappeller sensors, see (Mumm, 2002) for details. Headings that would cause the rappeller to move into unsafe regions are prohibited. Obstacle avoidance is a huge area of research and this is an extremely simplistic approach. This approach does 


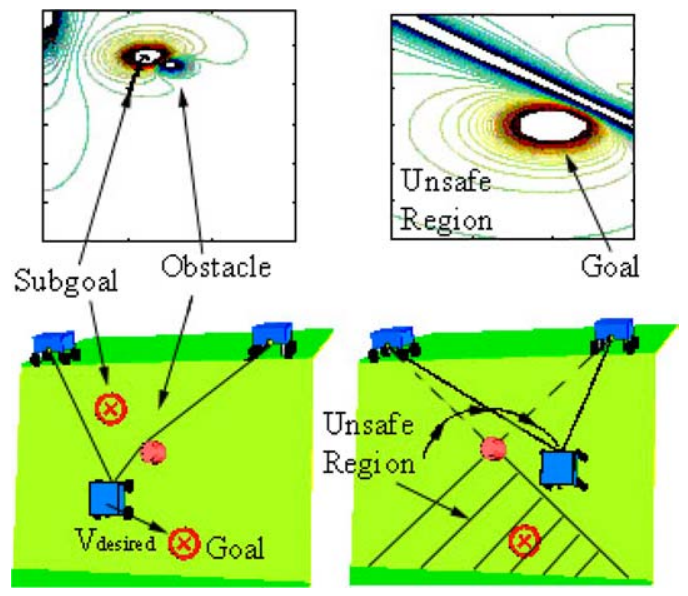

Figure 5. Avoid tether catch behavior.

not ensure optimality or that the target will be reached, however, it does ensure safety assuming an accurate map. Future work could include a more sophisticated method.

The Avoid Tether Catch behavior detects and prevents the tether from being caught on obstacles. Tether catches are detected, and obstacle location can be estimated, using tether angles at the rappeller and at the anchor. This behavior creates an unsafe region based on the approximate obstacle location, Fig. 5. A sub-goal is then placed between the obstacle and the anchor robot with the uncaught tether, Fig. 5 lower left. A negative potential is then assigned to the rappeller, obstacle, and anchors. A positive potential is placed at the sub goal and headings are chosen based on the resulting potential field. Example potential fields are shown in the upper figures in Fig. 5. This ensures the rappeller motion will relieve the tether catch while moving toward the sub goal. When the first sub goal is attained, other sub goals (not shown) are placed to lead the rappeller to the goal (Mumm, 2002). The unsafe region is retained in the workspace map. If a tether catch is not detected, this behavior's output is identical to the output of Motion to Goal.

The Motion to Goal behavior is on the lowest level. This behavior determines the position of the rover using tether angles (see Appendix) and produces a heading toward the goal.

\subsection{Action Selection}

A method is needed to determine which behavior(s) determine the action of the robots. Both command fusion and arbitration techniques are used. With command fusion, multiple behaviors contribute to the systems actions while arbitration techniques select one behavior to dictate the systems actions (Pirjanian, 2000).

There are three points in the behavior hierarchy where action selection occurs. At the lowest level the outputs of Maintain Safe Heading and Avoid Tether Catch are combined using fuzzy logic based command fusion. Both behaviors contribute to the output (safe rappeller \& anchor velocities) provided to the higher level. The action selection technique used "partially satisfies" each behavior (Pirjanian, 2000).

The outputs of Maintain Safe Heading and Avoid Tether Catch are first combined (multiplied) and the resulting function is "defuzzified" using a Center of Gravity method (Pirjanian, 2000; Mumm, 2002). For example, consider the system configuration shown in Fig. 6. Here the rappeller is at the edge of the workspace directly below the right anchor, therefore the output of Maintain Safe Heading prohibits headings $0^{\circ}<$ $\theta<180^{\circ}$. The output of Maintain Safe Heading is a multi-valued function of acceptable rappeller headings, Fig. 6 top right. A tether catch does not exist so the output of Avoid Tether Catch is identical to Motion to Goal. Since the goal is directly below the rappeller the output of Motion to Goal is a multi-valued function centered about a zero degree heading, Fig. 6 right middle. These functions are combined (multiplied) and the resulting heading is found using a center of gravity method, Fig. 6 right bottom. This example outputs a heading of -2.5 degrees while "sufficiently satisfying" (Pirjanian, 2000) both behaviors. This causes the rappeller to move toward the goal while staying safely in the workspace.

At the intermediate level an arbitration technique is used to determine the system's actions. The output of the command fusion described above, the Haul behavior, and the Avoid Singularities behavior are considered in the arbitration decision. The Avoid Singularities behavior takes priority, followed by the Haul behavior. If neither of these behaviors is active, the output of the above command fusion is passed to the Velocity Sync behavior.

At the highest level are the non-competitive behaviors Communicate and Velocity Syncs. The Communicate behavior occurs at a predetermined rate; otherwise, Velocity Sync is active.

There are system configurations where the aggregation of behaviors produces no acceptable rappeller 


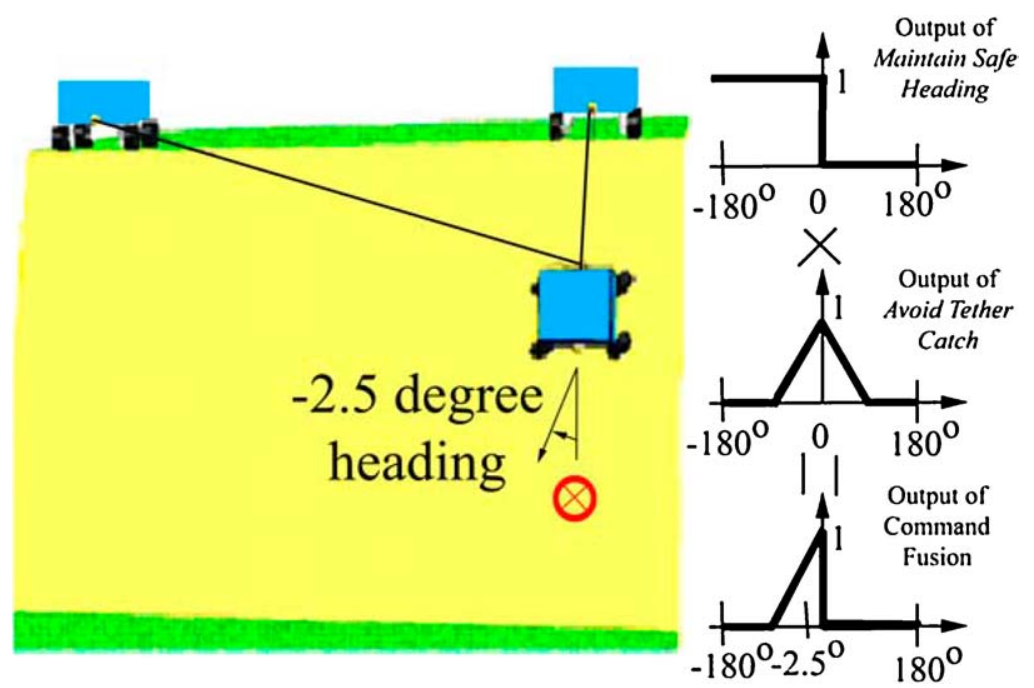

Figure 6. Command fusion of Maintain Safe Heading and Motion to Goal.

heading. For example, if the goal is outside the workspace. In this case the system will abort and wait for outside instruction.

\section{System Modeling and Controller Stability}

\subsection{System Modeling}

The physical system is highly non-linear. A model of the rappeller, Fig. 7, was created for stability analysis as well as the simulation analysis described below. The model represents the rappeller as a point mass with two degrees of freedom (the rappeller does not rotate). The tethers are modeled as spring/dampers that apply forces to the rappeller $\left(F_{R}\right.$ and $\left.F_{L}\right)$. A traction force acts in the rolling direction of the rappeller's wheels and a transverse force resists motion perpendicular to the wheels rolling direction. A simple frictional model is used to represent the complex soil/wheel interactions. For more on soil/wheel modeling for planetary rovers see (Iagnemma et al., 2002).

The non-linear state space equations that describe the rappeller motion are given by:
Where $k$ and $b$ are the stiffness and damping of the tethers and $m$ is the mass of the rappeller.

The system model was used to create a simulation of rappeller motion. The above model is written for the case where the tethers are in tension, however, the simulation accounted for the fact that the tethers cannot push (i.e. $F_{R, L}=0$ if $F_{R, L}<0$ ).

\subsection{Controller Stability}

The stability of the Velocity Sync control law was investigated. Nominal controller operation controls the tether winch speeds as a function of the rappeller velocity. The position of the rappeller is controlled (by lower level behaviors) by choosing rappeller velocities to move the rappeller to the goal. This analysis evaluates the stability of the Velocity Sync behavior.

Assumptions were made for the stability analysis, namely that the tethers are rigid and that the rappeller cannot produce traction (worst case, $F_{\text {traction }}=$ $F_{\text {transverse }}=0$ ). Both assumptions are valid if the rappeller is on a steep slope (large $\alpha$ ).

$$
\frac{\partial}{\partial t}\left[\begin{array}{c}
x \\
\dot{x} \\
y \\
\dot{y}
\end{array}\right]=\left[\begin{array}{c}
\dot{x} \\
-\frac{F_{R}}{m} \cos \left(\phi_{R}\right)-\frac{F_{L}}{m} \cos \left(\phi_{L}\right)+g \cos (\alpha)+\mu g \sin (\alpha) \cos (\theta)+\frac{F_{\text {transverse }}}{m} \cos (90-\theta)+\frac{F_{\text {traction }}}{m} \cos (\theta) \\
\dot{y} \\
-\frac{F_{R}}{m} \sin \left(\phi_{R}\right)-\frac{F_{L}}{m} \sin \left(\phi_{L}\right)+\mu g \sin (\alpha) \sin (\theta)+\frac{F_{\text {transverse }}}{m} \sin (90-\theta)+\frac{F_{\text {traction }}}{m} \sin (\theta)
\end{array}\right]
$$




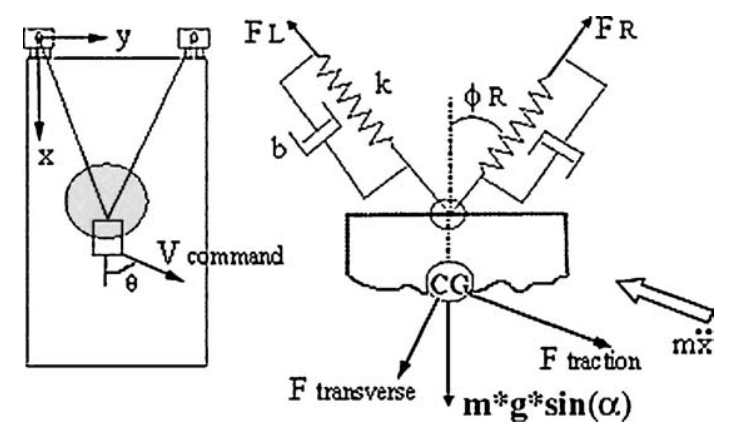

Figure 7. Rappeller model.

Assuming proportional control is used at the anchors to control the tether winch speeds, the tether forces are proportional to the difference between desired winch velocity and the actual winch velocity:

$$
\left[\begin{array}{c}
F_{L} \\
F_{R}
\end{array}\right]=\left[\begin{array}{c}
k\left(\dot{S}_{L, \text { desired }}-\dot{S}_{L, \text { actual }}\right) \\
k\left(\dot{S}_{R, \text { desired }}-\dot{S}_{R, \text { actual }}\right)
\end{array}\right]
$$

The desired winch velocity is given by the projection of the desired rappeller velocity onto the tether vectors (2). To evaluate the stability, the desired rappeller velocity is set to zero and (4) can be written in terms of the state vector (see Appendix):

$$
\left[\begin{array}{c}
F_{L} \\
F_{R}
\end{array}\right]=\left[\begin{array}{c}
k\left(0-\dot{S}_{L, \text { actual }}\right) \\
k\left(0-\dot{S}_{R, \text { actual }}\right)
\end{array}\right]=k\left[\begin{array}{c}
\frac{-x \dot{x}-y \dot{y}}{\sqrt{x^{2}+y^{2}}} \\
\frac{-x \dot{x}+(w-y) \dot{y}}{\sqrt{x^{2}+(w-y)^{2}}}
\end{array}\right]
$$

Since the Velocity Sync behavior controls rappeller velocity and not the rappeller position, the stored energy in the system is now given by:

$$
V=\frac{1}{2} x_{2}^{2}+\frac{1}{2} x_{4}^{2}=\frac{1}{2} \dot{x}^{2}+\frac{1}{2} \dot{y}^{2}
$$

The time derivative of the energy function is:

$\dot{V}=\frac{\partial V}{\partial x_{2}} \dot{x}_{2}+\frac{\partial V}{\partial x_{4}} \dot{x}_{4}=\frac{\partial V}{\partial \dot{x}} \ddot{x}+\frac{\partial V}{\partial \dot{y}} \ddot{y}=\dot{x}(\ddot{x})+\dot{y}(\ddot{y})$

Expressions for the $x$ and $y$ accelerations are given in (3) and can be substituted into (7) along with (5). Noting that $\phi_{R}$ and $\phi_{L}$ are functions of $x, y$ and the width of the workspace $w$ (see Appendix) the derivative of the energy function can be written, after much simplification, as:

$$
\begin{aligned}
\dot{V} & =-\frac{k(x \dot{x}+y \dot{y})^{2}}{m\left(x^{2}+y^{2}\right)^{1 / 2}}-\frac{k(x \dot{x}-(w-y) \dot{y})^{2}}{m\left(x^{2}+(w-y)^{2}\right)^{1 / 2}} \\
& =-\frac{k}{m}\left(F_{R}^{2}+F_{L}^{2}\right)
\end{aligned}
$$

This expression is negative for all values of position, velocity, and positive control gain $k$, and:

$$
\lim _{t \rightarrow \infty} \dot{V}=0
$$

Therefore, under the stated assumptions, the Velocity Sync control law is asymptotically stable for all positive control gains $k$.

\section{Sensitivity}

Field trials found that friction in the tether angle measurement mechanism can cause imprecise readings. The tether passes through a follower that is used to rotate an angular sensor. High (theoretically infinite) tether tension is required to move the follower to the perfect reading since the tether lacks transverse stiffness. Inherently, there are errors in the tether vector.

The controller's sensitivity with respect to tether vectors was investigated; in particular, the output of the Velocity Sync behavior was studied. Sensitivity was defined as the ratio between the angular error in the commanded rappeller velocity $(\delta \theta)$ and the angular error $\left(\delta \phi_{i}\right)$ in the $i$ th tether vector $\underline{T}_{i}$ where $i$ corresponds to either the Left or Right tether, see (10) and Fig. 8. The desired velocity is the input to the controller

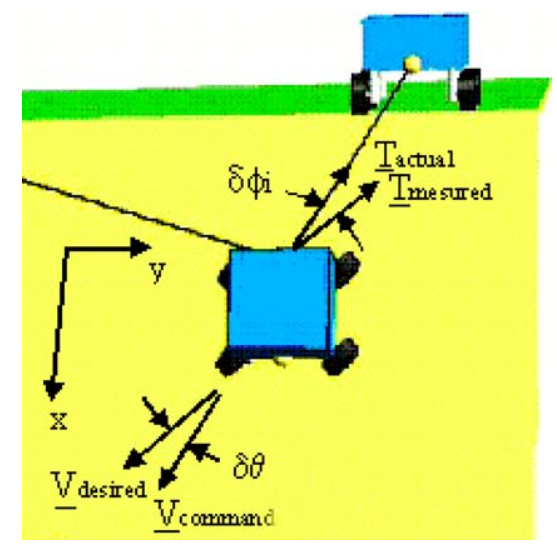

Figure 8 . Sensitivity analysis. 


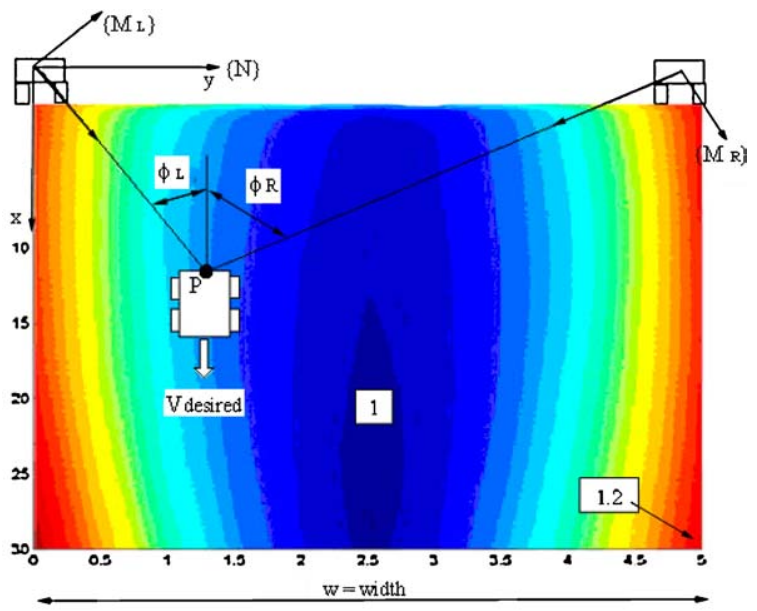

Figure 9. Workspace sensitivity for zero degree headings.

and the commanded velocity is the output of the controller.

$$
(\text { Sensitivity) })_{i} \equiv \frac{\delta \theta}{\delta \phi_{i}}
$$

To find the sensitivity an expression for $\underline{V}_{\text {command }}$ is required as a function of tether angles $\left(\phi_{L}\right.$ and $\left.\phi_{R}\right)$. The angle of $\underline{V}_{\text {command }}$ can then be compared to the angle of $V_{\text {desired }}$ to find the sensitivity. To find the rappeller velocity assign coordinate frames ( $\left\{M_{L}\right\}$ and $\left.\left\{M_{R}\right\}\right)$ to each tether with the origin at the anchor robot and the $x$-axis extending down the length of the tether, Fig. 9. Then an expression can be written for the velocity of the rappeller (point $P$ ) measured in the fixed frame $\{N\}$ relative to quantities expressed in the moving frame $\left\{M_{L}\right\}$, see (11).

$$
{ }^{N} \underline{V}_{P}={ }^{N} \underline{V}_{\text {Origin }, M_{L}}+\left(\underline{\omega}_{M_{L} / N} \times{ }^{M_{L}} \underline{R}_{P}\right)+{ }^{M_{L}} \underline{V}_{P}
$$

Where ${ }^{N} \underline{V}_{\text {Origin, } M_{L}}$ is the velocity of the origin of the $\left\{M_{L}\right\}$ frame measured in $\{N\}$ (i.e. the velocity of the left anchor robot); $\underline{\omega}_{M_{L} / N}$ is the angular velocity of the $\left\{M_{L}\right\}$ frame relative to $\{N\}$ (i.e. rotation of the tether); ${ }^{M_{L}} \underline{R}_{P}$ is the location of point $P$ in the $\left\{M_{L}\right\}$ frame (i.e. the tether length); ${ }^{M_{L}} \underline{V}_{P}$ is the velocity of point $P$ measured in the $\left\{M_{L}\right\}$ frame (i.e. winch speed).

A similar expression can be written for the velocity of point $P$ using terms expressed in the right moving frame $\left\{M_{R}\right\}$, see (12).

$$
{ }^{N} \underline{V}_{P}={ }^{N} \underline{V}_{\text {Origin }, M_{R}}+\left(\underline{\omega}_{M_{R} / N} \times{ }^{M_{R}} \underline{R}_{P}\right)+{ }^{M_{R}} \underline{V}_{P}
$$

The Velocity Sync behavior dictates the winch velocity $\left(\dot{s}_{i}\right.$ or $\left.{ }^{M_{i}} \underline{V}_{P}\right)$ given by

$$
\begin{aligned}
\dot{s}_{i} & ={ }^{M_{i}} \underline{V}_{P}=\underline{V}_{\text {desired }} \cdot T_{i} \\
& =V_{x} T_{x, i}+V_{y} T_{y, i}
\end{aligned}
$$

The winch velocity (13) can be substituted into (11) and (12), the tether length $\left({ }^{M_{L}} \underline{R}_{P}\right.$ and $\left.{ }^{M_{R}} \underline{R}_{P}\right)$ can be expressed in terms of the tether angles $\left(\phi_{L}\right.$ and $\left.\phi_{R}\right)$, and for this analysis the anchor robots are stationary $\left({ }^{N} \underline{V}_{\text {Origin }, M_{L}}={ }^{N} \underline{V}_{\text {Origin }, M_{R}}=0\right)$. Both (11) and (12) describe the velocity of the rappeller (point $P$ ). Equating these expressions yields a vector equation that can be solved for the two unknowns $\underline{\omega}_{M_{L} / N}$ and $\underline{\omega}_{M_{R} / N}$. Then (11) or (12) can be used to solve for the rappeller velocity.

Sensitivity depends on both the rappeller's location in the workspace and the desired heading. Sensitivity was evaluated throughout a 30 meter ( $x$ or down the cliff) by 5 meter ( $y$ or width) workspace for various headings.

The sensitivity for the rappeller moving at unit velocity on a zero degree heading is shown in Fig. 9. Here the commanded winch velocity is given by (14) where $\delta \phi_{i}$ is the sensor error.

$$
\begin{aligned}
\dot{s}_{i} & =\underline{V}_{\text {desired }} \cdot T_{i}=V_{x} T_{x, i}+V_{y} T_{y, i}=V_{x} T_{x, i} \\
& =(1) \cos \left(\phi_{i}+\delta \phi_{i}\right)
\end{aligned}
$$

Sensitivity is lowest in the bottom middle of the workspace and highest when the rappeller is below an anchor $\left(y_{\min }\right.$ and $\left.y_{\max }\right)$. Sensitivity also increases, and becomes undefined, near the top of the workspace near the anchors $\left(x=0\right.$ and $y=y_{\min }$ or $\left.y_{\max }\right)$. These areas are not shown in the figure because they overshadow all other areas. The center of the workspace has a sensitivity $\approx 1$ and increases to a maximum of 1.2 near the workspace edge. This shows that throughout the workspace errors in tether vectors will be directly translated to errors in rappeller velocity.

Figure 10 shows the sensitivity for ninety degree rappeller headings relative to error in the left tether vector. Here, sensitivity is lowest at the top $\left(x_{\min }\right)$ of the workspace and increases by a factor of five near the bottom $\left(x_{\max }\right)$. Sensitivity varies primarily in the $x$-direction. Therefore, at the bottom of the workspace $\left(\phi_{i}\right.$ approaches $\left.0^{\circ}\right)$ measurement errors in tether vectors will result in five times the error in rappeller velocity.

Similar analysis was performed for other headings and workspace dimensions. Several important 


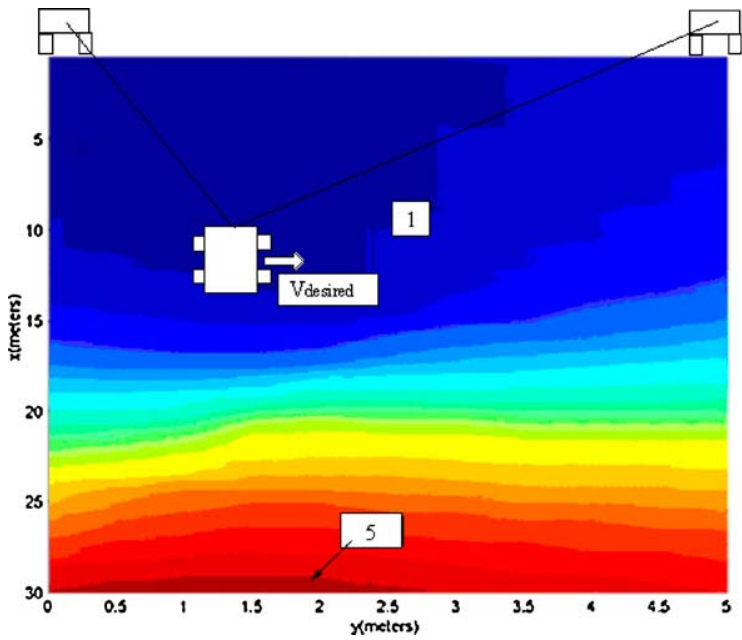

Figure 10. Workspace sensitivity for ninety degree headings.

conclusions can be drawn from these results. First, when descending and ascending the cliff $\left(\theta=0^{\circ}\right.$ and $\left.180^{\circ}\right)$, the rappeller should operate in the center of the workspace and wide workspaces should be avoided. This will ensure operation in areas of low sensitivity (bottom middle of Fig. 9). Second, for general rappeller motions (e.g., $\theta=90^{\circ}$ ) avoid narrow workspaces. This well keep tether angles high (i.e. $\phi_{i}$ not close to $0^{\circ}$ ) and the sensitivity low (upper half of Fig. 10).

These rules can be used to create future behaviors that coordinate anchor and rappeller motions. The anchors can be relocated to maintain low sensitivity based on desired rappeller motion.

\section{Test Studies}

\subsection{Laboratory Testing}

A laboratory system was created to evaluate controller performance, see Fig. 11. The test ramp (cliff face) has a slope of $60^{\circ}$ with a 1 meter by 2.5 meter workspace. The anchor robots were simulated using fixed winches with optical encoders to measure tether angles.

Several experiments were performed with the laboratory system. First, basic controller performance was verified for several headings at several locations in the workspace. Figure 12 shows a coordinated motion that moves the rappeller around a triangle for three cliff slopes $\left(\alpha=30^{\circ}, 45^{\circ}\right.$, and $\left.60^{\circ}\right)$. The desired path is shown as a solid line, the path created in simulation is shown as a dotted line, and the results from the laboratory system are shown as a dashed line. The rappeller

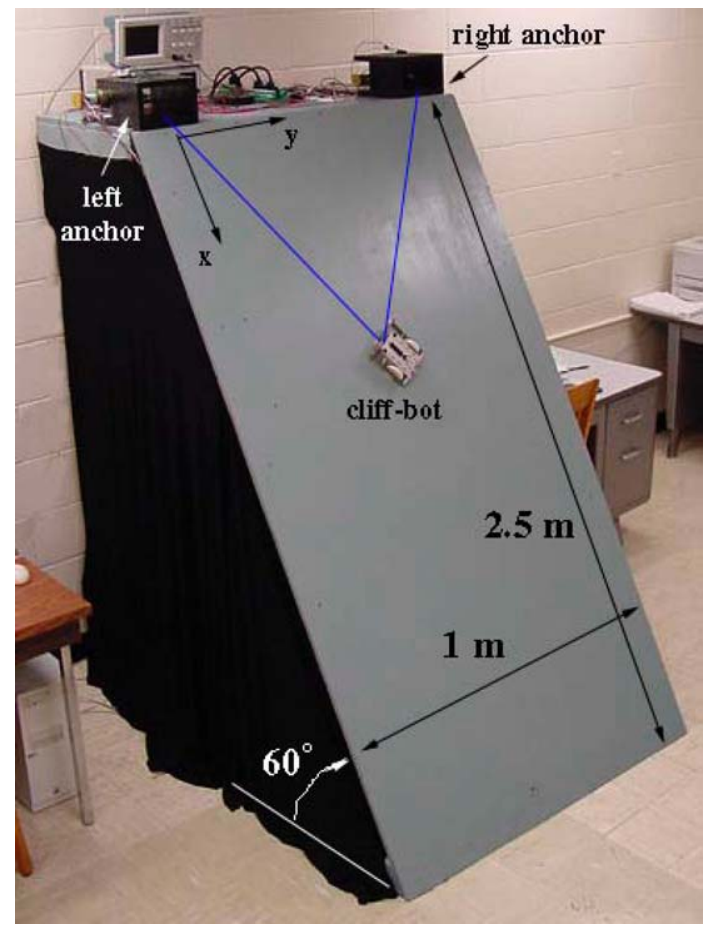

Figure 11. Laboratory experiments.

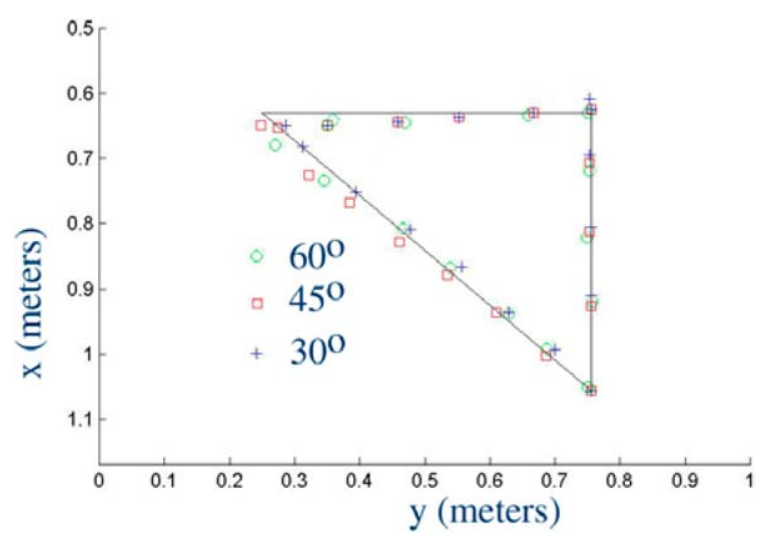

Figure 12. Laboratory experiments.

was given waypoints at each corner. After achieving a waypoint the rappeller stopped at each corner and turned in place. This traverse required heading angles of $90^{\circ}$ for $30 \mathrm{~cm}, 0^{\circ}$ for $40 \mathrm{~cm}$, and $-143^{\circ}$ for $50 \mathrm{~cm}$. For the laboratory system, the maximum deviation from the desired path was $3.6 \mathrm{~cm}$. The rappeller achieved each waypoint with a maximum error of $3.6 \mathrm{~cm}$ or $7.2 \%$ (of distance traveled).

Experiments were performed to verify the sensitivity analysis described in the previous section. In various 


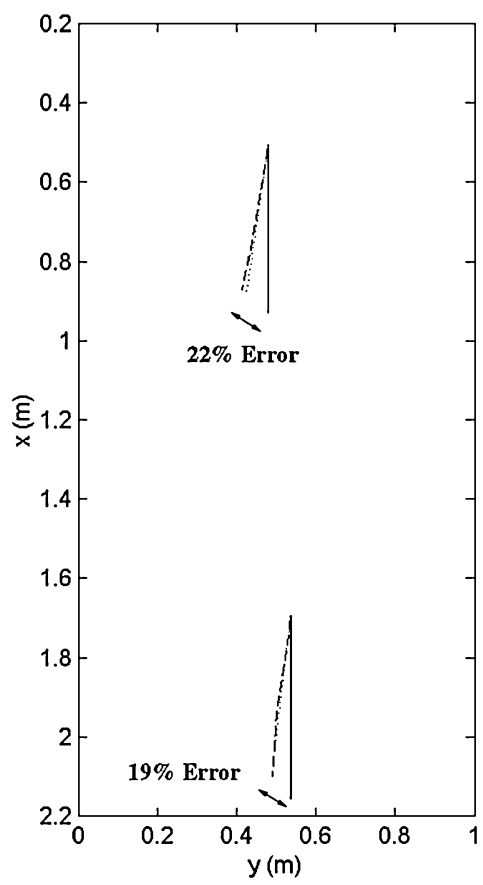

a) $0^{\circ}$ heading, left tether error

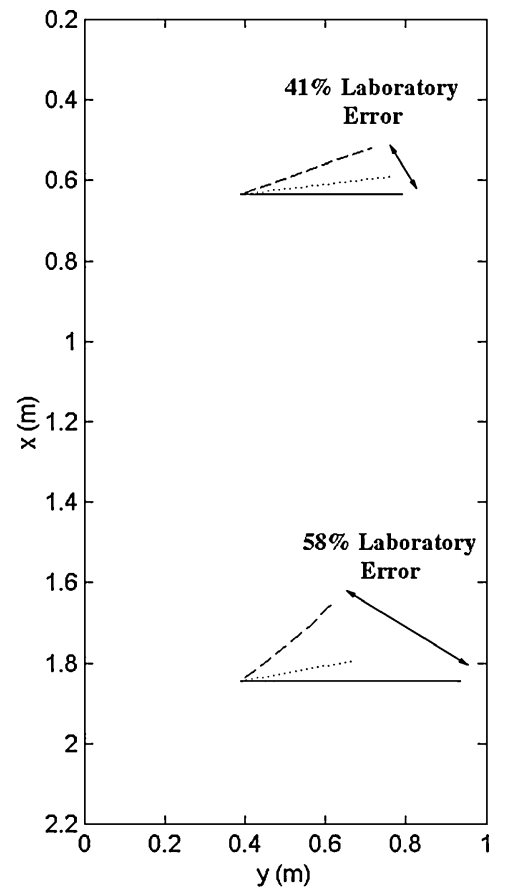

b) $90^{\circ}$ heading, left tether error

Figure 13. Laboratory conformation of sensitivity analysis.

experiments random and systematic errors were introduced into the tether angle sensors. The resulting errors in velocity synchronization produced significant errors in positioning accuracy, Fig. 13. Again, the desired path is shown as a solid line, the path from simulation is shown as a dotted line, and the results from the laboratory system are shown as a dashed line. Two traverses are shown in each figure (one higher and one lower in the workspace).

Figure 13(a) shows a $40 \mathrm{~cm}-0^{\circ}$ traverse (down the cliff) with errors in the left tether angle measurement given by $0^{\circ} \leq \delta \varphi_{L} \leq 10^{\circ}$. This error contains both random error that could result from electrical noise and systematic error that could result from sensor friction. Both laboratory (dotted line) and experimental results (dashed line) are shown. The high traverse resulted in a final position error of $22 \%$ while the lower traverse had $19 \%$ error. These results confirm the sensitivity analysis that predicted: (1) higher velocity synchronization errors higher in the workspace (wide workspace, $\varphi_{i} \approx 45^{\circ}$ ), and (2) small variation in errors throughout the workspace (error ration $=22 / 19=1.1$ ).

Figure 13(b) shows a $40 \mathrm{~cm}-90^{\circ}$ traverse with errors in the left tether angle measurement $\left(-10^{\circ} \leq \delta \varphi_{L} \leq\right.$ $\left.10^{\circ}\right)$. The high traverse resulted in $41 \%$ error while the lower traverse had 58\% error. These results also confirm the sensitivity analysis that predicted: (1) higher errors lower in the workspace (narrow workspace, $\varphi_{i}$ small), and (2) a dependency on location in the workspace (error ratio $=58 / 41=1.4$ ). This traverse shows a significant discrepancy between laboratory and

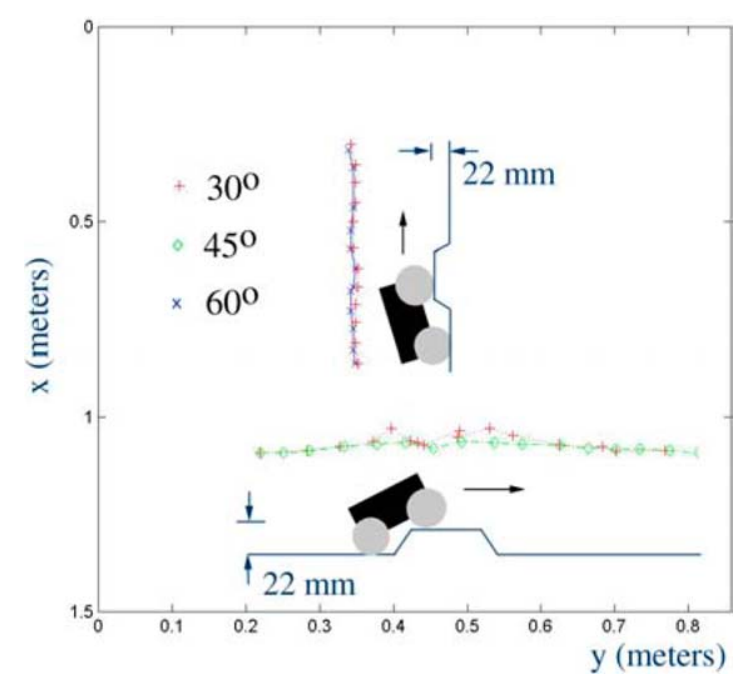

Figure 14. Rappeller over an obstacle. 


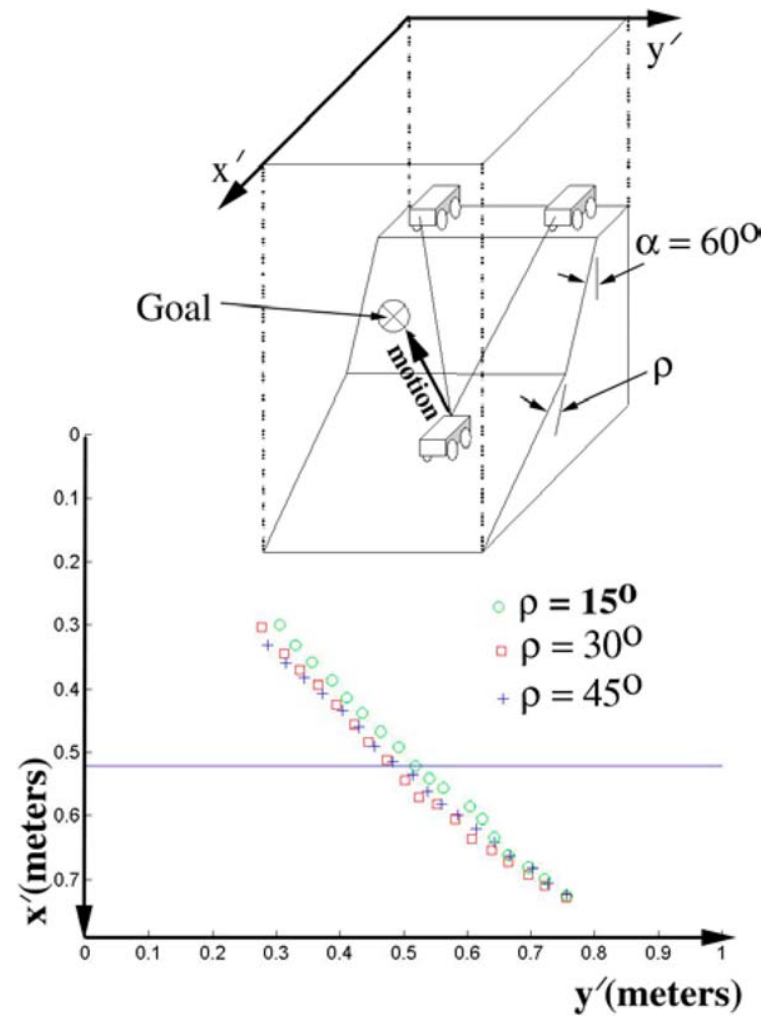

Figure 15. Change in slope.

simulation resulting from the simplified model of the transverse wheel forces.

The laboratory experiments confirmed the theoretical analysis and simulation results. Namely, when ascending or descending the cliff $\left(0^{\circ}\right.$ traverse $)$ maintain a narrow workspace $\left(\varphi_{i}\right.$ small $)$ and for general motions (e.g., $90^{\circ}$ traverse), maintain a wide workspace $\left(\phi_{i} \approx 45^{\circ}\right)$.

Other experiments were performed to study the effect of obstacles in the path of the rappeller. The rappeller was commanded to move in a straight line motion over an obstacle with a height that is $40 \%$ of the wheel diameter. The obstacle was a regular trapezoid in cross section with sides at $60^{\circ}$ to the base. This motion was repeated on cliff slopes $(\alpha)$ of $30^{\circ}, 45^{\circ}$, and $60^{\circ}$ in both vertical (up the cliff) and horizontal (across the cliff) directions, Fig. 14.

It can be seen that the rappeller performs well in motion up the cliff because the tethers are more directly assisting the rappeller motion. The largest error occurs during horizontal motion at smaller slopes $\left(30^{\circ}\right)$. In this case the rappeller receives little help from the tethers. This case approaches the "unconnected" condition where the rappeller is moving on its own.

Finally, experiments were performed where the rappeller moved across a change in cliff slope. These experiments were repeated for a vertical motion and a diagonal motion for changes in slope of $15^{\circ}, 30^{\circ}$, and $45^{\circ}$, Fig. 15 . Here, the measurements of both tether angles $(\phi$ and $\beta$ ) are required. The rappeller navigated these transitions and accuracy did not depend on the magnitude of the change.

\section{Summary and Conclusions}

The paper described a control system for a team of three robots to access cliff walls at inclines up to $70^{\circ}$. Two robot assistants lower a third rappeller robot down the cliff using tethers. The anchors use actively controlled winches to assist the rappeller in navigating the cliff face and retreating to safe ground. All three robots function as a team to explore the cliff face.

Three stability requirements were defined for maintaining system safety: (1) Maintain tension in each tether, (2) Avoiding dangerous tether configurations, and (3) Avoiding tether singularities. Behaviors were then defined to obtain systems goals such as maintaining safety and moving to desired waypoints. An action selection method was presented that uses both command fusion (multiple contribution) and arbitration (single contribution) to determine the actions of the system. The action selection method uses both discrete event states as well as fuzzy logic based command fusion.

An analysis of the control system's stability and sensitivity to measurement errors in tether angles was performed. The stability analysis showed that, under the stated assumptions, the Velocity Sync control law is asymptotically stable for all positive control gains. The sensitivity analysis suggested rules to minimize tracking errors in the presence of noisy tether angle measurements. For ascending and descending the cliff the anchor robots should remain close together. For general motions across the cliff face the anchor robots should remain spaced so tether angles are relatively high $\left(\approx 45^{\circ}\right)$.

Laboratory and field tests were presented that quantified controller performance and confirmed the results of the sensitivity analysis. 


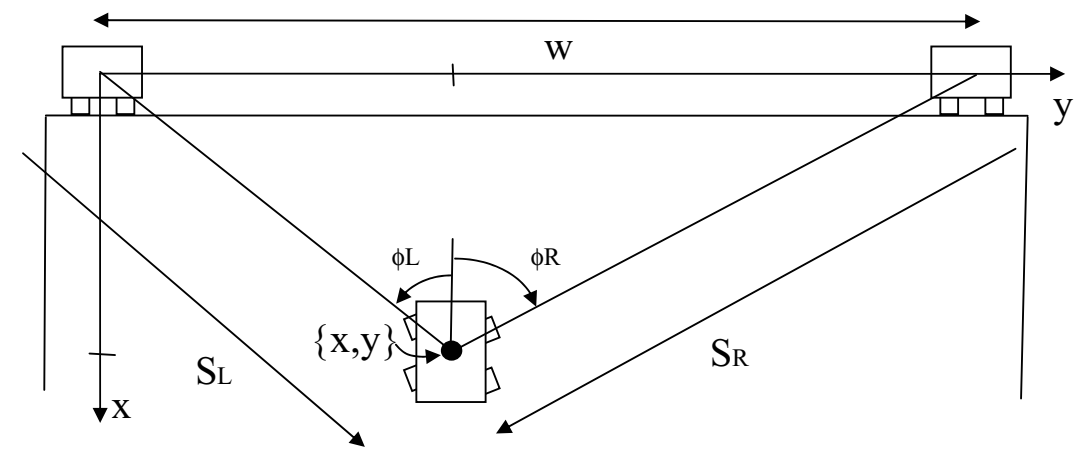

Figure 16. System geometry.

\section{Appendix A}

Tethers in tension of lengths $S_{L}$ and $S_{R}$ are related to the rappeller position $\{x, y\}$, Fig. 16 .

The relationship is given by:

$$
\begin{aligned}
& S_{L}=\sqrt{x^{2}+y^{2}} \\
& S_{R}=\sqrt{x^{2}+(w-y)^{2}}
\end{aligned}
$$

The tether angles are related to the rover position by:

$$
\begin{array}{ll}
\cos \left(\phi_{L}\right)=\frac{x}{\sqrt{x^{2}+y^{2}}} & \cos \left(\phi_{R}\right)=\frac{x}{\sqrt{x^{2}+(w-y)^{2}}} \\
\sin \left(\phi_{L}\right)=\frac{y}{\sqrt{x^{2}+y^{2}}} & \sin \left(\phi_{R}\right)=\frac{w-y}{\sqrt{x^{2}+(w-y)^{2}}}
\end{array}
$$

Equation (15) can be used to express the tether velocity terms of the rappeller coordinates:

$$
\begin{aligned}
& \dot{S}_{L}=\frac{\partial}{\partial t}\left(\sqrt{x^{2}+y^{2}}\right)=\frac{x \dot{x}+y \dot{y}}{\sqrt{x^{2}+y^{2}}} \\
& \dot{S}_{R}=\frac{\partial}{\partial t}\left(\sqrt{x^{2}+(w-y)^{2}}\right)=\frac{x \dot{x}-(w-y) \dot{y}}{\sqrt{x^{2}+(w-y)^{2}}}
\end{aligned}
$$

Expressions (16) and (17) can be used to write the tether velocity as functions of tether angles:

$$
\begin{aligned}
\dot{S}_{L} & =\left(\frac{x}{\sqrt{x^{2}+y^{2}}}\right) \dot{x}+\left(\frac{y}{\sqrt{x^{2}+y^{2}}}\right) \dot{y} \\
& =\cos \left(\phi_{L}\right) \dot{x}+\sin \left(\phi_{R}\right) \dot{y} \\
\dot{S}_{R} & =\left(\frac{x}{\sqrt{x^{2}+(w-y)^{2}}}\right) \dot{x}-\left(\frac{w-y}{\sqrt{x^{2}+(w-y)^{2}}}\right) \dot{y} \\
& =\cos \left(\phi_{R}\right) x-\sin \left(\phi_{L}\right) y
\end{aligned}
$$

These expressions are identical to the control law described for the Velocity Sync behavior.

\section{Acknowledgments}

The authors would like to acknowledge the work of Tony Gannino, Brett Kennedy, Hrand Aghazarian, Mike Garrett, and Lee Magnone in the design and construction of the field system. This research was partially performed while Erik Mumm was a visiting researcher in the Planetary Robotics Laboratory of the Jet Propulsion Laboratory, California Institute of Technology, under a contract with the National Aeronautics and Space Administration.

\section{References}

Apostolopoulos, D. and Bares, J. 1995. Locomotion configuration of a robust rappelling robot. In Proceedings of the 1995 IEEE/RSJ International Conference on Intelligent Robots and Systems, Human Robot Interaction and Cooperative Robots (IROS '95), vol. 3.

Arkin, R. 1987. Motor schema based navigation for a mobile robot: An approach to programming behavior. In IEEE International Conf. on Robotics and Automation, pp. 264-271.

Arkin, R. 1998. Behavior Based Robotics, The MIT Press.

Brooks, R. 1986. A robust layered control system for a mobile robot. IEEE Journal of Robotics and Automation, RA-2(1):14-23.

Farritor, S., Hacot, H., and Dubowsky, S. 1998. Physics-based planning for planetary exploration. In IEEE International Conference on Robotic and Automation.

Fuskushima, E.F., Kitamura, N., Hirose, S. 2001. Development of tethered autonomous mobile robot system for field works. $A d$ vanced robotics, 15(4):481-496.

Gat, E., Desai, R., Ivlev, R., Loch, J., and Miller, D. 1994. Behavior control for robotic exploration of planetary surfaces. IEEE Trans. on Robotics and Automation, 10(4). 
Gorman, J., Jablokow, K., and Cannon, D. 2001. The cable array robot: Theory and experiment. In IEEE International Conference on Robotics and Automation, Seoul, Korea.

Huntsberger, T., Pirjanian, P., and Schenker, P. 2001. Robotic outposts as precursors to a manned Mars habitat. In Proc. Space Technology and Applications International Forum.

Iagnemma, K., Shibly, H., and Dubowsky, S. 2002. On-line terrain parameter estimation for planetary rovers. In IEEE Intl. Conference on Robotics and Automation, Washington, DC.

Khatib, O. 1986. Real-time obstacle avoidance for manipulators and mobile robots. International Journal of Robotics Research, 5(1):90-98.

Latombe, J. 1991. Robot Motion Planning, Kluwer Academic Publisher.

Mumm, E. 2002. Behavior-based control for cooperative robotic planetary cliff descent. Masters Thesis, Department of Mechanical Engineering, University of NebraskaLincoln.

Murphy, R. 2000. An Introduction to AI Robotics, The MIT Press.

Pirjanian, P. 2000. Multiple objective behavior-based control. Journal of Robotics and Autonomous Systems, 31(1/2): 53-60.

Pirjanian, P., Huntsberger, T., Trebi-Ollennu, A., Aghazarian, H., Das, H., Joshi, S., and Schenker, P. 2000. CAMPOUT: A control architecture for multirobot planetary outposts. In SPIE, vol. 4196.

Saffioti, A., Ruspini, E., and Konolige, K. 1997. Using fuzzy logic for mobile robot control. Handbook of Fuzzy ts and Possibility Theory, Kluwer Academic.

Schenker, P., Huntsberger, T., Pirjanian, P., Trebi-Ollennu, A., Das, H., Joshi, S., Aghazarian, H., Ganino, A., Kennedy, B., and Garrett, M. 2000a. Robot work crews for planetary outposts: Close cooperation and coordination of multiple mobile robots. In Proc. SPIE, vol. 4196.

Schenker, P., Pirjanian, P., Balaram, B., Ali, K., Trebi-Ollennu, A., Huntsberger, T., Aghazarian, H., Kennedy, B., Baumgartner, E., Iagnemma, K., Rzepniewski, A., Dubowsky, S., Leger, P., and Apostolopoulos, D. 2000b. Reconfigurable robots for all terrain exploration. In SPIE, vol. 4196.

Seraji, H., Howard, A., and Tustel, E. 2001. Safe navigation on hazardous terrain. In IEEE International Conference on Robotics and Automation, Seoul, Korea.

Shiang, W., Cannon, D., and Gorman, J. 1999. Dynamic analysis of the cable array robotic crane. In IEEE International Conference on Robotics and Automation, Detroit, MI, USA.

Rosenblatt, J., Williams, S., and Durrant-Whyte, H. 2000. Behaviorbased control for autonomous underwater exploration. In Proc. IEEE Intl. Conf. on Robotics and Automation, pp. 920-925.

Volpe, R., Baumgartner, E., Schenker, P., and Hayati, S., 2000. Technology development and testing for enhanced Mars rover sample return operations. In IEEE Aerospace Conference.

Weisbin, C., Rodriguez, G., Schenker, P., Baumgartner, E., Volpe, R., Hayati, S., and Das, H. 1999. Autonomous rover technology for Mars sample return. In Proc. I-SAIRAS '99 (5th International Symposium on Artificial Intelligence, Robotics and Automation in Space).

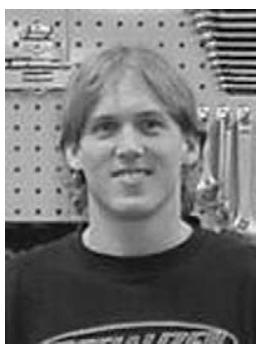

Erik Mumm is a Project Engineer for Honeybee Robotics in New York City. He earned his BSME in May 2000 and his MSME in May 2002 from the University of Nebraska-Lincoln. During his Masters research he worked alongside NASA/JPL engineers in the Planetary Robotics Lab at JPL. Erik's other interests include basketball, Nebraska baseball and the Mets.

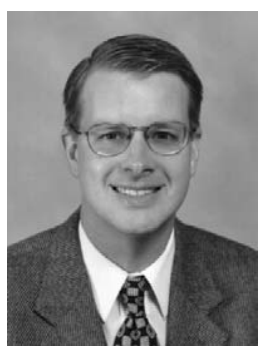

Shane Farritor is an Assistant Professor of Mechanical Engineering at the University of Nebraska-Lincoln. His research interests include space robotics, surgical robotics, biomedical sensors, and robotics for highway safety. He holds courtesy appointments in both the Department of Surgery and the department of Orthopeadic Surgery at the University of Nebraska Medical Center. He serves of both the AIAA Space Robotics and Automation technical committee and ASME Dynamic Systems and Control Robotics Panel. He received M.S. and Ph.D. degrees from M.I.T.

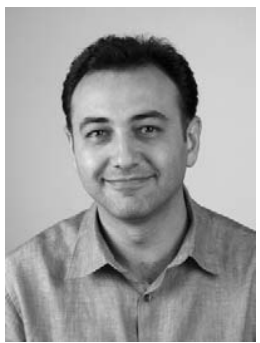

Paolo Pirjanian is Chief Scientist at Evolution Robotics, Inc. His recent research and development consists of vision-based localization and mapping and state-of-the-art robotic control architecture. Prior to joining Evolution Robotics, he was a lead engineer at the Jet Propulsion Laboratory working on robotics technologies for space exploration. Results of his work have been featured on CNN TV, CNN.com, NASA TV, and other media. Paolo is also a part-time faculty member at the Computer Science department of University of Southern California, where he teaches graduate-level artificial intelligence. He has authored many articles and book chapters on 
numerous subjects within the fields of robotics, computer vision, artificial intelligence, and virtual reality. Paolo has also served on organizing committees for various technical conferences in the US and Europe and serves as a reviewer for technical publications. Paolo received the M.Sc.CS. and the Ph.D. degrees in 1994 and 1998, respectively, both from Aalborg University, Denmark.

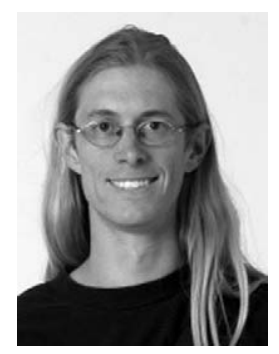

Chris Leger after realizing he was unlikely to visit Mars in person, Chris Leger decided that the next best thing would be to travel there vicariously through a robot. With that in mind, he received a BS in Computer Engineering from Carnegie Mellon in 1995, and M.S. and Ph.D. degrees in Robotics from CMU in 1997 and 1999. He now works on the Mars Exploration Rover mission at the Jet Propulsion Laboratory. His other passions include rock climbing, playing bass, luthiery, and writing.

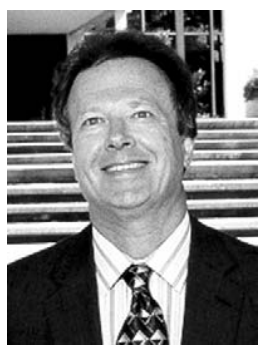

Paul S. Schenker is Manager, Mobility Systems Concept Development Section, Jet Propulsion Laboratory (JPL), California Institute of Technology, Pasadena, and as such, responsible for JPL R\&D in planetary mobility and robotics. His published research includes topics in robotic perception, robot control architectures, telerobotics and teleoperation, multi-sensor fusion, and most recently, multi-robot cooperation. He has led the development of robotic systems that include the Field Integrated Design \& Operations Rover (FIDO), Planetary Dexterous Manipulator (MarsArm, microArm), Robot Assisted Microsurgery System (RAMS), Robotic Work Crew (RWC), and All Terrain Explorer (ATE/Cliff-bot), with resulting technology contributions to NASA missions. Dr. Schenker is active in the IEEE, Optical Society of America, and SPIE. He has served as an elected Board member and 1999 President of the last; he currently serves as an elected member of the NAS/United States Advisory Committee to the International Commission for Optics. 